\title{
MONOCLONAL GAMMOPATHIES IN A SERIES OF 1743 PLASMA DONORS
}

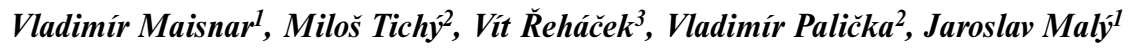

Charles University in Prague, Faculty of Medicine and University Hospital in Hradec Králové: II $^{\text {nd }}$ Department of Medicine - Department of Clinical Haematology ${ }^{1}$, Institute of Clinical Biochemistry and Diagnostics ${ }^{2}$, Department of Transfusion Medicine ${ }^{3}$

Summary: We carried out a retrospective analysis on a series of 1743 plasma donors during the period from January 1999 to February 2004 to determine the frequency of monoclonal gammopathies occurrence. In the whole group, we observed $0.68 \%$ occurence and in the subgroup of donors over 40 years of age even $1.18 \%$ occurrence of the serum paraprotein. We have presented our recommendations for the screening of monoclonal gammopathies in blood donors.

Key words: Monoclonal gammopathy, Blood and plasma donors, Electrophoresis

\section{Introduction}

Monoclonal gammopathies (MG) are a very heterogeneous group of disorders characterized by the presence of a monoclonal immunoglobulin (paraprotein) in the serum which can pass into the urine (1). This abnormal monoclonal protein is produced by a single clone of plasma cells which are the terminal stage of B-lymphocyte proliferation. The main difference between monoclonal and polyclonal immunoglobulins is a homogeneity of their polypeptide chains (6). Monoclonal gammopathies are classified as malignant monoclonal gammopathies and monoclonal gammopathies of unknown or undetermined significance (MGUS) $(1,8,9)$. The most frequent and clinically significant malignant monoclonal gammopathy is multiple myeloma.

MGUS are much more frequent than malignant monoclonal gammopathies and their occurrence is age dependent. The prevalence of paraproteinemias is about $1 \%$ in people up to the age of 60 and about $10 \%$ in persons older than 80 years of age (10). We found only sporadic studies in the literature describing the occurrence of monoclonal gammopathies in a series of blood or plasma donors $(3,4)$. In these studies - most of which are over 15 years old - immunoelectrophoresis is used for the identification of monoclonal immunoglobulin. This method is less sensitive than the immunofixation used today (7). We expected a higher occurrence of monoclonal immunoglobulins because we have used better methods for establishing them. We studied the population of plasma donors, where the electrophoresis was set as a screening investigation before the actual donation (5).

\section{Material and methods}

We examined blood serum of 1743 plasma donors (1197 men and 546 women, 18-60 years of age) by electrophoretic analysis during the period from January 1999 to February 2004. Electrophoresis was done twice a year at six-monthly intervals. The total protein was determined by biuret method (ROCHE) on the Hitachi 914 device. We used the Hydrasis (SEBIA) equipment for the electrophoretic analysis. The quantity of paraproteins was determined by densitometric method on the Hyris (SEBIA) equipment.

\section{Results}

A total of 7311 electrophoretic analyses were done in the period of observation. The immunofixation was performed in $72(4.1 \%)$ plasma donors because of the suspicion of paraprotein being present in their blood serum. In 12 donors we found a different quantity of monoclonal immunoglobulin: $1.8-14.42 \mathrm{~g} / \mathrm{l}$. The paraprotein of $\mathrm{IgG}$ class was observed in 8 donors (6x kappa and $2 x$ lambda), the paraprotein of IgA class was found in 2 donors (1x kappa and $1 \mathrm{x}$ lambda), paraproteinemia IgM-kappa in one case and double paraproteinemia IgG-kappa+IgA-kappa was proved in another one (overview in Table 1). In the whole group of all 1743 plasma donors, we observed a paraprotein occurrence of $0.68 \%$. If we subdivided the studied group into two age groups, the group of people up to 40 years of age (981 donors), would show $0.30 \%$ and the group of people over 40 years of age $1.18 \%$ occurrence (in 762 donors). All donors, except for No. 5 with transient paraproteinemia, were eliminated from the blood donors register and were sent to 
the Department of Clinical Hematology in our hospital for observation. The case of donor No. 10 with paraprotein IgA-lambda hidden in beta-globulin region during electrophoresis was very alarming. Only after the examination of a pathological rib fracture the multiple myeloma was diagnosed in this patient.

\section{Discussion}

The number of monoclonal gammopathies has increased in the last few years. There are numerous reasons. The main factor is that we have been using more sensitive laboratory methods and concurrently there is the evidence of higher age-limit $(9,2)$. Monoclonal gammopathies are quite a rare diagnosis in young people, we confirmed this fact in our study by finding only three monoclonal gammopathies in the group of donors under 40 years of age. This frequency $(0.3 \%)$ correlates with results of other authors $(3,4)$. However, the frequency observed in the group of donors over 40 years of age is not negligible because of the fact, that MGUS is considered precancerous today. It is well known, that over the years, relatively a high proportion of MGUS will transform into the malignant monoclonal gammopathies. Kyle and others described this transformation in $16 \%$ of MGUS patients over 10 years, and in $40 \%$ over 25 years of their monitoring $(10,12)$

The occurrence of monoclonal gammopathies $1 / 145$, as we established in our study of 1743 plasma donors, is not insignificant. The best screening test for the proof of M-gradient is electrophoresis on an agarose gel, or capillary electrophoresis which is even better. Using these methods we are able to prove the presence of monoclonal proteins in a concentration of about $0.5 \mathrm{~g} / \mathrm{l}$. However, the determination of monoclonal gradient hidden in beta-globulin region is always problematic to find, as it was identified in one of our patients. A similarly difficult situation occurs when only the monoclonal free-chains kappa or lambda are present, and the M-gradient may not be obvious by electro- phoresis of serum. The proof of protein in urine by test papers is not sufficient enough, because it is based especially on the determination of albumin (11). The protein in urine had to be examined by a standard test with sulfosalicylic acid. If positive, we have to use the electrophoresis, and, if there is a suspicion of the presence of monoclonal protein, we have to do immunofixation subsequently.

In our opinion, all blood donors, not only plasma donors, have to undergone an electrophoretic serum examination at least once a year, and if this test proves to be positive, then it is necessary to do an immunofixation for the exclusion or the confirmation of monoclonal protein presence. It would be ideal to perform immunofixation on all donors annually, the screening immunofixation by pentavalent serum should be sufficient. This screening test would be beneficial for both, donor (detection of monoclonal gammopathy and their consequential monitoring) and recipient of blood preparations at the same time.

\section{References:}

1. Alexanian R, Weber D, Liu F. Differential diagnosis of monoclonal gammopathies. Arch Pathol Lab Med 1999;123:108-113.

2. Cohen JH, Crawford J, Rao MK et al. Racial differences in the prevalence of monoclonal gammopathy in community-based sample of the elderly. Amer J Med 1998; 104:439-444

3. Fine JM, Fine A, Lambin P. Dépistage systématique des gammapathies monoclonales dans une population de 3.758 sujets de race noire (Sénégal). Rev Fr Transfus Immunohématol 1977;20:309-313.

4. Fine JM, Marneux M. Donnees recentes sur les gammapathies monoclonales. Rev Fr Transfus Immunohématol 1985;28:591-600.

5. Guide to the preparation, use and quality assurance of blood components. $9^{\text {th }}$ ed. Strasbourg: Council of Europe Publishing, 2003.

6. Keren DF. Procedures for evaluation of monoclonal immunoglobulins. Arch Pathol Lab Med 1999;123:126-132.

7. Keren DF, Alexanian R, Goeken JA et al. Guidelines for clinical and laboratory evaluation of patients with monoclonal gammopathies. Arch Pathol Lab Med 1999;123:106-107.

8. Kyle RA. Monoclonal gammopathy of undetermined significance and solitary plasmocytoma. Hematol Oncol Clin North Am 1997;11:71-87.

9. Kyle RA, Rajkumar SV. Monoclonal gammopathies of undetermined significance. Hematol Oncol Clin North Am 1999;13:1181-1202.

10. Kyle RA, Therneau TM, Rajkumar SV et al. A long-term study of prognosis in monoclonal gammopathy of undetermined significance. N Engl J Med 2002; 346:564-569.

Tab. 1.

\begin{tabular}{|c|c|c|c|c|c|c|c|}
\hline \multirow{2}{*}{ Donor } & \multirow{2}{*}{ Sex } & Age & Type of PP & Quantity of PP & \multirow{2}{*}{ Diagnosis } & \multicolumn{2}{|c|}{ Number of donations } \\
& & Whole blood & Plasma \\
\hline 1 & F & 44 & IgG-kappa & 11.1 & MGUS & 16 & 1 \\
\hline 2 & F & 57 & IgG-kappa + IgA-kappa & $4.63+2.8$ & MGUS & 45 & 61 \\
\hline 3 & M & 31 & IgG-lambda & 11.31 & MGUS & 7 & 123 \\
\hline 4 & M & 47 & IgA-kappa & 7.78 & MGUS & 48 & 61 \\
\hline 5 & M & 31 & IgG-kappa & 1.9 & MGUS & 10 & 83 \\
\hline 6 & M & 32 & IgG-kappa & 2.07 & not examine & 7 & 43 \\
\hline 7 & F & 54 & IgM-kappa & 1.8 & not examine & 20 & 97 \\
\hline 8 & M & 49 & IgG-kappa & 3.68 & MGUS & 20 & 1 \\
\hline 9 & F & 42 & IgG-lambda & 9.31 & MGUS & 14 & 2 \\
\hline 10 & M & 57 & IgA-lambda & 6.23 & MM & 31 & 83 \\
\hline 11 & F & 45 & IgG-kappa & 14.42 & MGUS & 14 & 1 \\
\hline 12 & M & 55 & IgG-kappa & 1.82 & MGUS & 24 & 66 \\
\hline
\end{tabular}

PP ... paraprotein; MM ... multiple myeloma 
11. Levinson SS. Urine protein electrophoresis and immunofixation electrophoresis supplement one another in characterizing proteinuria. Ann Clin Lab Sci 2000; 30:79-84

12. Vuckovic J, Ilic A, Knezevic N et al. Prognosis in monoclonal gammopathy of undetermined significance. Brit J Haematol 1997;97:649-651

Submitted October 2005.

Accepted May 2006.

MUDr. Vladimír Maisnar, Ph.D.,

University Hospital,

IInd Department of Medicine

- Department of Clinical Hematology,

Sokolská 581,

50005 Hradec Králové, Czech Republic.

e-mail: maisnar@fnhk.cz 